\title{
Automatic and efficient metallic surface defect detection based on key pixel point locations
}

\author{
Jiahui Yu, Hongwei Gao, Jian Sun, Wei Yang, Yueqiu Jiang, and Zhaojie Ju
}

\begin{abstract}
Surface defect detection aims to accurately recognize and distinguish types of defects and plays a key role in many applications. However, most of the recent studies focus on specific scenario detection and do not fairly consider the balance between the speed and accuracy. In the paper, we propose a key pixel points location-oriented method to identify multiscale defects, with several important properties: 1) A real-time template matching-based model is designed to speed up the process by introducing the Gaussian operator; 2) An improved Hough-based model is used to achieve a higher detection precision by deep mining both incremental properties and parallel properties; 3) An adaptive filtering-based image preprocessing method is proposed to eliminate the interference of multiple types of clutters and noises. In the experiments, a mean average rate of $96 \%$ was achieved to detect and classify four types of common defects and the average time was reduced to $0.149 \mathrm{~s}$. Furthermore, we fully evaluate the proposed method on two public datasets collected in real production lines and compare the results with other state-of-the-art methods. The results show that the proposed method achieved better balanced performance in many real application scenarios.
\end{abstract}

Index Terms - defects detection, pixel detector, Gaussian operator, incremental Hough transform, imaging denoising

\section{Introduction}

$\mathrm{S}_{\mathrm{in}}^{\mathrm{u}}$ URFACE defect detection is a key process for the assembly in many industrial applications, such as circuit assembly, PCB detection, steel production, and semiconductor industry [1] [2]. With the development of surface measurement techniques in machine vision, surface defect detection has gained impetus over the last ten years [3]. The low efficiency and high cost of

The authors would like to acknowledge support from the following projects: LiaoNing Province Higher Education Innovative Talents Program Support Project (Grant No. LR2019058); LiaoNing Revitalization Talents Program (Grant No. XLYC1902095); National Natural Science Foundation of China (Grant No. 51575412, 51575338, U1609218 and 51575407); CAS Interdisciplinary Innovation Team (Grant No. JCTD-2018-11); EU INTERREG Project AiBle; DREAM project of EU FP7-ICT (Grant No. 611391).

Corresponding authors: Zhaojie Ju; Hongwei Gao.

Jiahui Yu, Hongwei Gao, Jian Sun, Wei Yang, Yueqiu Jiang are with the school of Automation and Electrical Engineering, Shenyang Ligong University, Shenyang, 110159, China (e-mail: ghw1978@sohu.com, yujiahui77@163.com, jiayou2017sj@163.com, xcfyangwei@163.com, yueqiujiang@sylu.edu.cn).

Zhaojie Ju is with the School of Computing, University of Portsmouth, Portsmouth, PO1 3HE, UK (e-mail: zhaojie.ju@port.ac.uk). conventional manual inspection cannot meet the requirements of high-quality inspection. For automatic and accurate detection, machine vision systems have been widely applied in many studies that have achieved excellent results. In general, surface defects detection methods consist of four types, that is, spectra, statistical, model-based methods, and deep learning-based methods. A new spectra-based system was designed for fabric defect detection by applying the thermal camera, which achieved excellent results [4]. The key of these methods is to select the ideal filter. Wang et al. proposed a new statistical-based model for bolt connection, which extended Hough-based transform line detection [5]. Similarly, these types of methods are widely studied, such as co-occurrence matrix and histogram methods [6] [7]. For model-based methods, more complex data and intensive calculation are required [8]. However, these studies have four main limitations: 1) strongly affected by light; 2) sensitive to data noise; 3 ) large amount of calculation and slow matching speed; 4) deviation of matching accuracy. Noted that most existing computer vision-based methods are proposed for special surface defect detection. Defect detection is usually aimed at certain types of products, detecting product defects caused by production errors and mistakes in the production process. The product material, production environment, and its own parameters are different, so the defect detection system focuses on address the particular problem rather than widespread problems.

In recent years, motivated by the success of neural networks, such as Convolutional Neural Networks (CNNs) and Recurrent Neural Networks (RNNs), many deep learning-based systems have emerged and been the powerful technology for surface defect detection. Most state-of-the-art deep models aim to improve feature extraction. A high-level feature learning model was proposed by Zou et al. for cracks detection, known as DeepCrack, which achieved end-to-end detection [9]. An effective BP-based feature learning network was proposed for robust and discriminative representation extraction [10]. For defective feature extraction, a gray level co-occurrence matrix was proposed by extending the k-nearest neighbor algorithm [11]. In many studies, model input and parameter training are also the key research directions. Sun et al. proposed a CNN-based network for adaptive multiscale image extraction, known as AMIC, which introduced pre-adoptive training on ImageNet [12]. Besides, Chang et al. proposed a new deep model and extended an optimization signal design algorithm, which used reflected signals as model input instead of images [13]. However, deep learning-based methods still face many challenges. First, considerable samples must be collected and labeled for training deep models. In this process, special expensive equipment and a lot of time and effort usually be 
required, which increases system designed costs. Furthermore, the specially trained model would be far from satisfactory on other datasets.

To solve the problems mentioned above, we propose a more applications, including scratch, crack, missing, and needling. For discriminative feature extraction, we design targeted discretize the Hough parameter space feature extractor. From the perspective of model construction, both the incremental property and the parallel property are deeper mined to obtain a compact model. The main contributions are summarized as follows.

1) A Gaussian operator is introduced into the pyramid-based model to achieve a real-time defect detection.

2) An effective image preprocessing method for eliminating four types of common image noise is proposed with a strong generalization ability.

3) A powerful edge detection method is proposed enable the model to resist noise interference and retain clearer edge features.

4) We propose to locate surface defect points at the pixel level, namely, the coordinates of each point in circles and lines, rather than the entity level, to better characterize defects.

The remainder of this paper is organized as follows. Section II briefly reviews related work about the hardware system choices. Section III describes the proposed defect detection model in detail. Section IV reports the dataset collection and results analysis. Section V concludes the paper and gives the future work.

\section{Related Work}

\section{A. Feature extraction}

Surface defect detection is a great challenge in the real application. The main reason is that most defect detection problems need to be resolved by the custom-made solutions. For extracting the discriminative features, in recent years, hand-crafted features methods and deep learning-based methods have emerged and achieved state-of-the-art results [14] [15]. For hand-crafted features methods, Li et al. proposed an X-ray-based detection system that extended 2D wavelet transform methods [16]. Yazdchi et al. proposed a new multifractal-based machine vision system by combining the hand-fractal method and the neural network [17]. Besides, With the development of machine learning, neural networks, such as CNNs and RNNs, have been greatly advanced. Many excellent new architectures and strategies that achieve a wide range of industrial applications are shown as follows. Cai et al. proposed a novel deep learning-based method for SMT (surface-mount technology) solder joint detection by introducing a cascaded CNN [18]. A novel local binary pattern-based feature extraction method was proposed by Yildiz et al. for fine fibers detection, which achieved the objective, easy, rapid, time, and cost-effective results [19]. Liu et al. proposed a structured multi-feature extraction method for spatiotemporal activity recognition, which is an inspiration for defect detection [20].

\section{B. Computer vision system}

In many studies, such as [21] and [22], the selection and calibration of multiple types of sensors are considered to be key general computer vision-based system for metal surface defect detection. The proposed method can achieve excellent and balanced results on four types of general defects in many action

issues. The key sensors consist of industrial camera, lens, light source and image acquisition card. In [23], a light source-based system was proposed that consisted of red and blue light and a line scan camera. The basic idea is to analyze the differences between both light and introduce texture and morphology attributes-based feature extraction model. Both light source calibration method and multilayer perceptron model are the key technology for the study. Similarly, in [24], a deep learning system consists of an underwater camera, and a robotic arm scanner was proposed for the crack and non-crack surface detection. Many novel technologies, including four convolutional layers, a Naive Bayes classifier, and a data fusion scheme were extended in the study. Besides, in [25], a new bionic system based on phase deflectometry method was proposed which consisted of a flexible robotic arm and a structured light projector.

In addition, stereoscopic vision systems (SVS) and laser-based methods have been developed in recent years. First, SVS-based methods can reconstruct digital scenes for target image recognition and match by using a group of cameras/sensors [26]. For example, a novel SVS-based system was proposed to improve the accuracy of three-dimensional distance measurement, in which a neural network was used to train optimal parameters [27]. Next, the camera-based vision system can be replaced by laser coordinates measurement and surface reconstruction techniques. For example, a novel robot vision system was designed to compute the average three-dimensional coordinates of the target, in which the laser dynamic triangulation technique was used effectively [28]. Similar, Artificial Neural Network (ANN) was also an effective method for triangulation [29].

\section{Problems and challenges}

Surface defects detection aims to analyze many types of defects that are defined by human experience in real applications. Normally, most of the studies focus on three problems, that is, the specific type of defects, the location of defects, and the size of defects. The effective system should achieve excellent results in these three areas. The following challenges need to be addressed:

1) In the industrialized world, very few defect images can be collected which is challenging to develop the computer vision system, including deep learning-based methods and traditional algorithm-based methods. Although the data synthesis method, such as mirroring, rotation, translation, and distortion, can be used to increase the number of defect samples, it is a large gap between the real data and the synthesized data.

2) Most defect detection methods focus on improving the recognition rate and classification accuracy, but the recognition speed receives little attention in many studies. It is challenging to analyze defects in real-time under the premise of ensuring detection accuracy. To solve this problem, both speed up model algorithms and hardware needs to be improved.

3) The causes of defects are complex. For example, defect 
features are difficult to extract from complex backgrounds, the defect scale changes greatly and the types are various, illumination changes are complex, and irregular surface reflections usually occur [30] [31] [32]. It is challenging to develop generalization systems to identify different defects. To solve this problem, data preprocessing
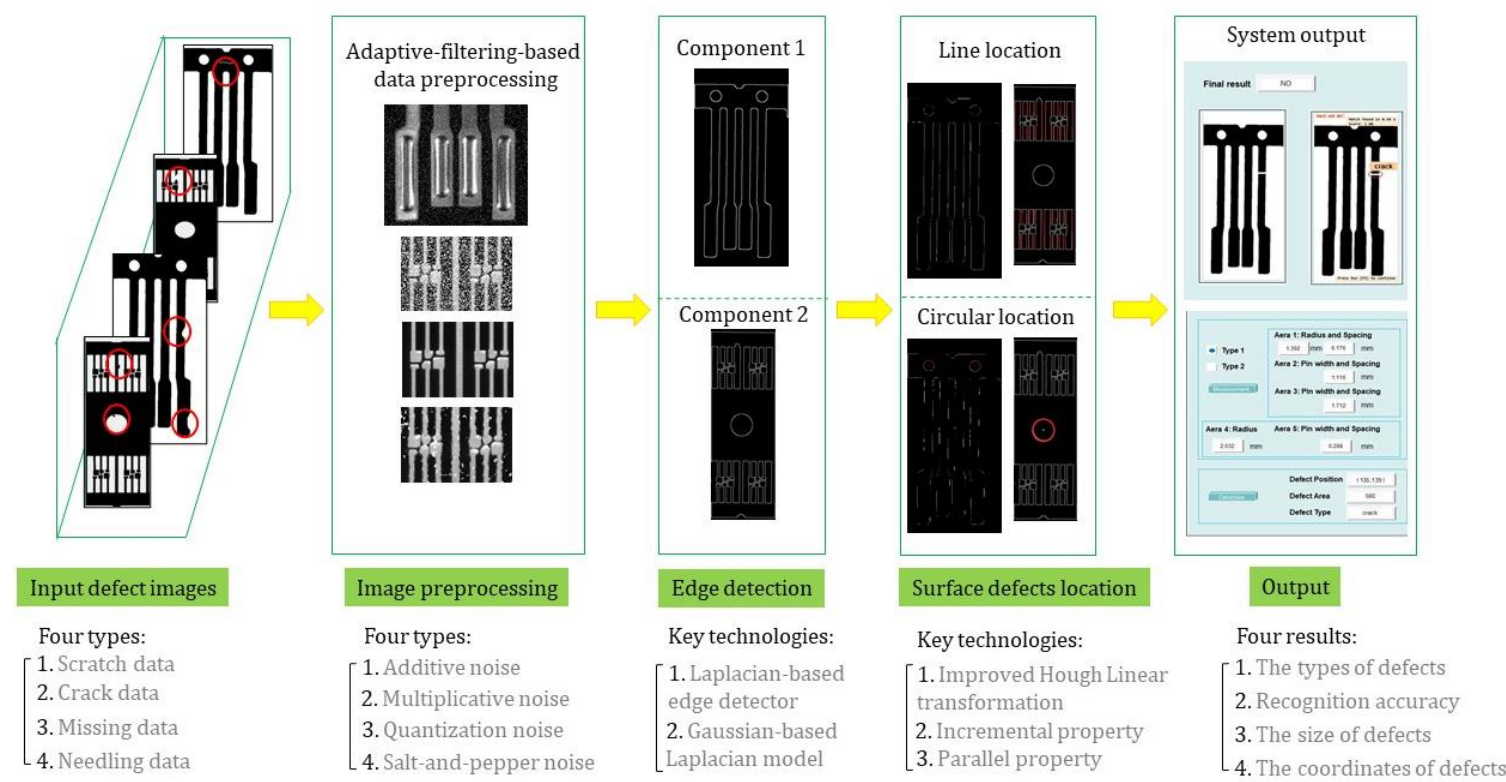

Fig.2. Flow chart of the proposed method

methods and defect detection and recognition algorithms need to be improved.

\section{Proposed Framework}

In the paper, the machine vision system consists of a CMOS (Complementary Metal-Oxide Semiconductor) camera, two light sources, a trigger system, and an image acquisition card, as shown in Fig. 1. Specifically, the procedure followed is the next: 1) The CMOS camera consists of four parameters, that is, the pixel size matrix is $3072 * 2048$, framerate is $17 \mathrm{fps}$, exposure time is in the $[27 \mu \mathrm{s}, 2.5 \mathrm{~s}]$, and the pixel size is $2.4 \mu \mathrm{m}$; 2) The lens model is MVL-HF1628M-6MP; 3) Considering the illumination uniformity, LED (Light-Emitting Diode) is best for our study and the placement angle is $45^{\circ}$ (Note that the dark field illumination technique can also be applied effectively, such as the 3D laminar flow-based method in [33]); 4) The image acquisition card model is MV-8808 because of its excellent compatibility; 5) The external trigger system includes I / O (Input/Output), motion control, level conversion unit, etc. A Flow-chart of the proposed system is shown in Fig.2, this framework is described as follows: 1) The framework consists of five parts, that is, input defect images, data preprocessing, edge detection, defects location, and output results; 2) The raw images with different defects are as the input; 3) For data preprocessing, four types of noises can be eliminated effectively, including common noises and special noises; 4) To detect the edge of metal components, both Laplacian-based edge detector and Gaussian-based Laplacian method are proven to perform well; 5) Surface defects location is the key technology in this proposed system, and its basic idea is to mine incremental properties and parallel properties; 6) Finally, detection results can be outputted, including the types of defects, recognition accuracy, the size of defects, and the coordinates of defects. Additionally, our main goal is to detect two types of metal components which consist of five areas, these detection areas are shown in Fig. 3 and the design dimensions diagram of metal components is shown in Fig. 4.

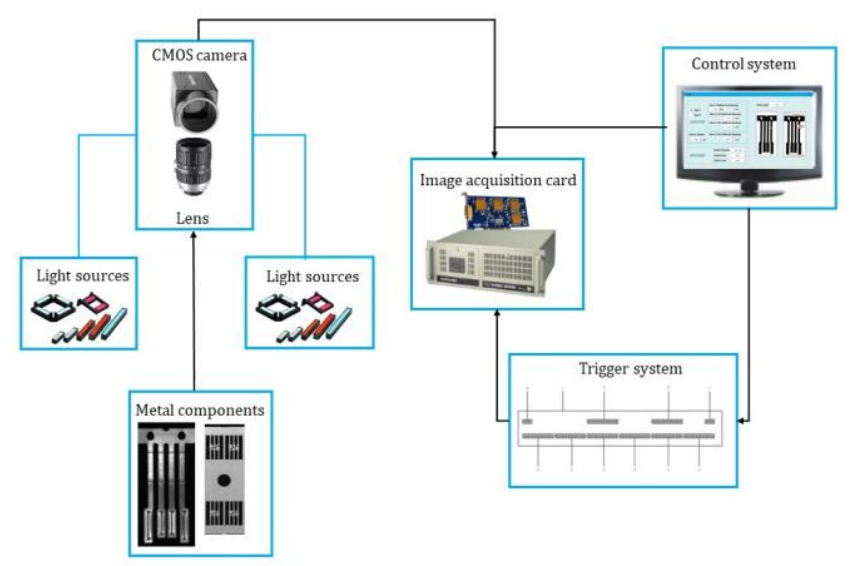

Fig.1. Block diagram of the machine vision system

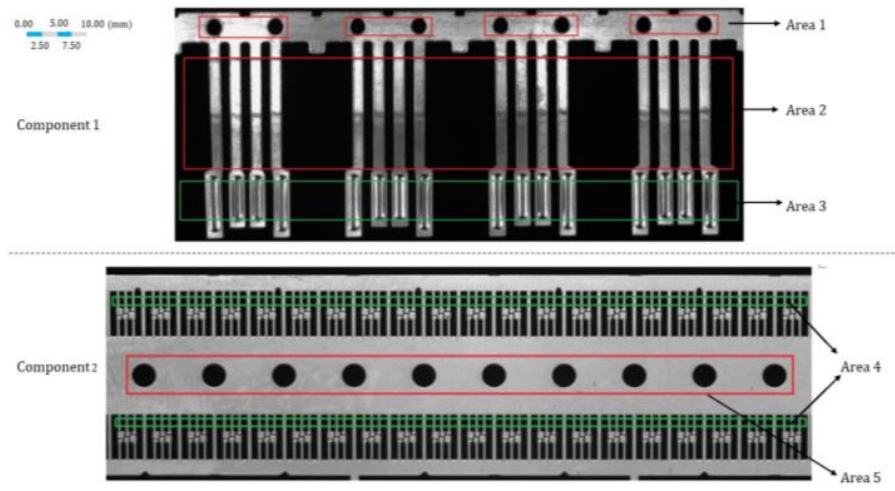

Fig.3. Two types of metal components with five detection areas. 
When the position of the camera is fixed, we conduct the camera calibration for optimizing image clarity. Accuracy is the key index in the image acquisition, as shown in (1). Where the working distance $W D$ is $8.7 \mathrm{~cm}$, the size of lens $s$ is $9 \mathrm{~mm}$, the back focal distance $F L$ is $16 \mathrm{~mm}$, and the depth of field is ignored. After the calibration process, the accuracy $\sigma$ is 0.016 . $\sigma=\frac{\operatorname{ss}(W D / F L)}{L}=\frac{48.9375}{3072} \approx 0.016 \mathrm{~mm} /$ pixel

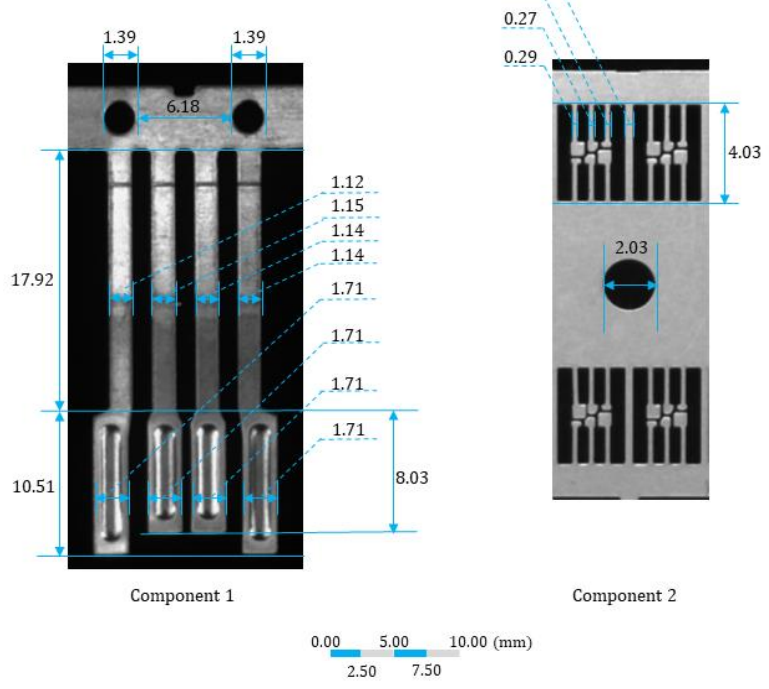

Fig.4. Design dimensions diagram of metal components.

\section{A. Edge detection}

Edge data with discriminative features is crucial in terms of online surface defect detection. To detect the edge of the object more accurately, edge-finding based methods and zero-crossing based methods have been proposed for keeping more representative edge data, in which the former detects the edge position by calculating the maximum value of the first-order differential and the latter finishes it by calculating the zero point of the second-order differential. Compared to the first-order differentiation based methods, the latter has more advantages: 1) More sensitive to grey value mutation so that can position the image edge more accurately and steadily; 2) Rotation-invariant so that can eliminate the influence of edge gradient changes. However, simply using the second-order differential based method might have a negative influence on sharpening and edge detection. More importantly, the edge data reduction may result in excessive noise. To alleviate this issue, we utilize the following edge detection method.

First, we customize a kind of Laplacian-based edge detector, which is tailored for metal surface inspection scene. To make it clear, we briefly review the concept of Laplacian. As an edge detector, Laplacian has a stronger ability to localize image edge, it is defined as (2), where $f(i, j)$ is two-dimensional discrete data.

$$
\begin{aligned}
& \nabla^{2} f(x, y)=\frac{\partial^{2} f}{\partial x^{2}}+\frac{\partial^{2} f}{\partial y^{2}} \\
& \frac{\partial^{2} f}{\partial x^{2}}=(f(i+1, j)-f(i, j))-(f(i, j)-f(i-1, j)) \\
& =f(i+1, j)+f(i-1, j)-2 f(i, j) \\
& \frac{\partial^{2} f}{\partial y^{2}}=(f(i, j+1)-f(i, j))-(f(i, j)-f(i, j-1)) \\
& =f(i, j+1)+f(i, j-1)-2 f(i, j)
\end{aligned}
$$

After (2), the (3) for data sharpening can be obtained.

$$
\begin{aligned}
& \nabla^{2} f= \\
& {[f(i+1, j)+f(i-1, j)+f(i, j+1)+f(i, j-1)]-4 f(i, j)}
\end{aligned}
$$

The Laplacian has the same detection results for the image rotated in all directions. Therefore, the Laplacian is extremely reductive to the edges of the data. Additionally, the application of Laplacian shortens the time required for edge detection. This is because, compared with the first-order-based method, it avoids the multiple calculation steps of convolution before summing.

Furthermore, to reduce the impact of edge data noise and keep more detailed data after image edge sharpening, we propose a Gaussian-based Laplacian model which is more robustness for discrete noise data analysis, as shown in (4).

$$
\Delta\left[G_{\delta}(x, y) * f(x, y)\right]=\left[\Delta G_{\delta}(x, y)\right] * f(x, y)=L o G * f(x, y)
$$

After the Gaussian model calculation, the data is processed as follows:

$$
\frac{\delta^{2}}{\delta^{2} y} G_{\sigma}(x, y)=\frac{y^{2}-\sigma^{2}}{\sigma^{4}} e^{-\left(x^{2}+y^{2}\right) / 2 \sigma^{2}}
$$

We can obtain the Gauss-Laplace convolution kernel by combining the above equations, as shown in (6).

$$
\begin{aligned}
L o G & \cong \Delta G_{\sigma}(x, y)=\frac{\partial^{2}}{\partial x^{2}} G_{\sigma}(x, y)+\frac{\partial^{2}}{\partial y^{2}} G_{\sigma}(x, y) \\
& =\frac{x^{2}+y^{2}-2 \sigma^{2}}{\sigma^{4}} e^{-\left(x^{2}+y^{2}\right) / 2 \sigma^{2}}
\end{aligned}
$$

As shown in Fig. 5, the edge results of two types of metal components by applying the proposed method and other state-of-the-art methods. Compared with other methods, the proposed model can get less noise and clearer data.
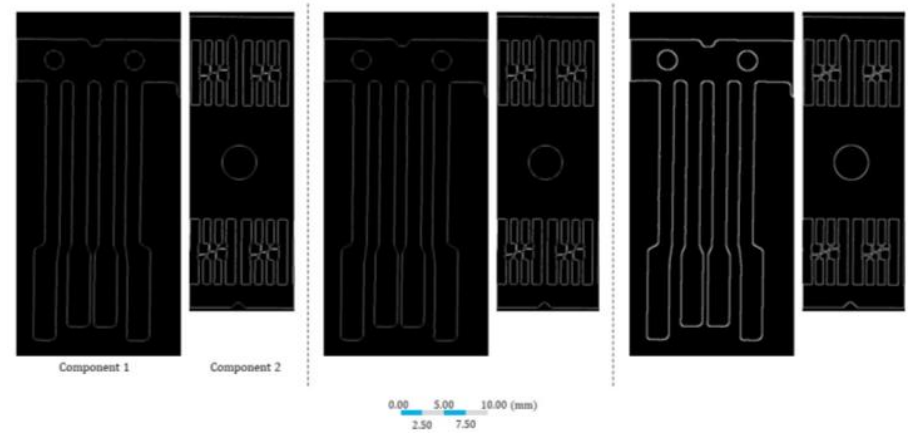

Fig. 5. Edge detection. From left to right is the Sobel-operator-based method, Prewitt-based method, our proposed method. For visualization, the rail image is not preprocessed.

\section{B. Surface defects location}

After image edge detection, defects location in the image should be measured more accurately. Although the shape of the defects is complex, their areas consist of circles and lines. 
Sub-pixel-based method is one of the most well-known tools for surface defect area detection. Despite the progress in the development of measurement accuracy, the time of measurement remains in the major limit. Therefore, in the paper, while improving the accuracy of point location, we reduce the delay and achieve real-time detection. To improve the detection accuracy, we proposed two sub-pixel-based methods for circular and line location, respectively (as shown in Fig. 7).

For line location (as shown in the left part of Fig. 7), we proposed the improved Hough linear transformation by introducing the iterative step and removing each iteration points, which consists of the following steps: 1) Discretize the points in all lines on the surface of the metal component, as shown in (7); 2) Perform Hough transform on all discretized points; 3) Finding the most representative points (these points make up set $\mathrm{X}$ ) in the line by using 2D accumulator; 4) Finding the set $\mathrm{Y}$ from the set $\mathrm{X}$ so that the distance to the line is less than the unit distance, as shown in $(8)$; 5) The points in the set $Y$ are combined into the optimal line by using the least square method; 6) Finding the points in the set X near the optimal line, and then remove them from the accumulator; 7) Iterating the above steps until the number of points in set $\mathrm{X}$ is less than the set value; 8) The return value after the termination of the algorithm is the line distance. In the proposed method, all the points are subjected to Hough transform only twice, including constructing the array by using the $2 \mathrm{D}$ accumulator and removing parts of points from the accumulator.

$$
\begin{gathered}
x_{i}-c \rightarrow \vec{x}_{i} \\
c=0.5\left(\begin{array}{l}
x_{\max }+x_{\min } \\
y_{\max }+y_{\min }
\end{array}\right) \\
\|\vec{x}-(\vec{a}+k \vec{b})\| \leq d x \text {, and } k=\langle\vec{b}, \vec{x}-\vec{a}\rangle
\end{gathered}
$$

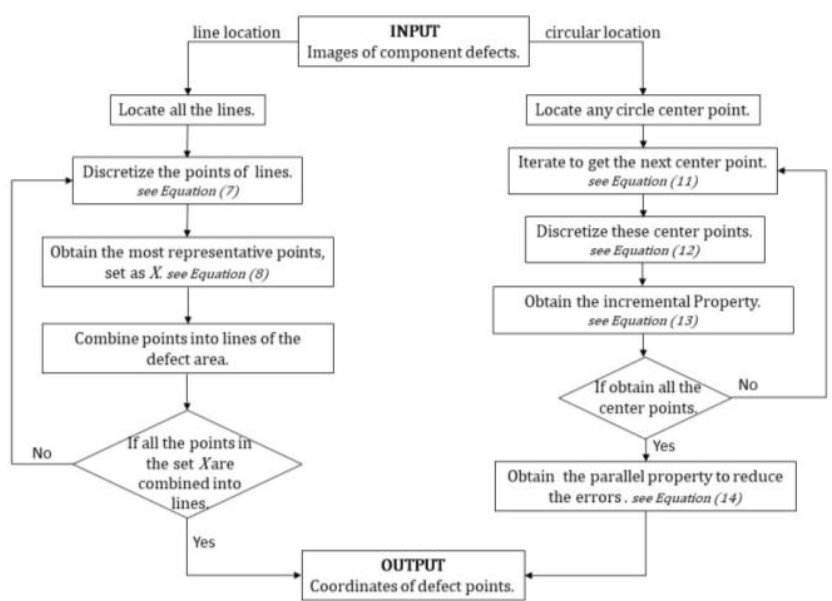

Fig.6. Flow chart of the iterative process, including the line location (left part) and the circular location (right part).

For circular location (as shown in the right part of Fig. 7), we introduced the incremental property and the parallel property to improve the Hough transform method. Specifically, the former can save the resources required for calculation to reduce the cost of hardware, and the latter can directly reduce the time required for calculation that can achieve the real-time detection. The equation of the circle is shown in (9) and the parametric representation of the circle is shown in $(10)$, where $(a, b)$ is the

center and $r$ is the radius. When using the traditional method to calculate circle size, more cost of operation can be greatly increased because of involving a large number of mathematical conversion and trigonometric functions. For simplicity, we proposed a new voting process by reducing the use of trigonometric functions in the Hough transform method, the detailed process was shown as follows.

$$
\begin{gathered}
(x-a)^{2}+(y-b)^{2}=r^{2} \\
\left\{\begin{array}{c}
x=a+r \cos \theta \\
y=b+r \cos \theta
\end{array}\right.
\end{gathered}
$$

The basic idea of the incremental property is to reuse the previous center points to obtain the next points, as shown in (11). Specifically, the value $\left(a_{n+1}, b_{n+1}\right)$ at the iteration $n+1$ can be obtained from the previous value $\left(a_{n}, b_{n}\right)$ at the iteration $n$. Discretized values are shown in (12), where $n$ is the angle index, $n_{\theta}$ is the angle resolution, $\lambda$ is the number of angle values. First, the $\sin \theta$ and $\cos \theta$ are approximated, and then the angle values are rearranged to obtain the incremental property, as shown in (13). The advantages of the proposed method are summarized as follows: 1) No trigonometric function for operating simply; 2) Linear incremental function for reducing approximation errors; 3) The basis of the parallel property because $a_{n}$ and $b_{n}$ can be calculated independently.

$$
\begin{aligned}
& \quad\left(a_{n+1}, b_{n+1}\right)=f\left(a_{n}, b_{n}\right) \\
& a_{n}=a+r \cos \theta_{n}, \theta_{n}=n \lambda \text { and } 0 \leq \theta_{n}<2 \\
& b_{n}=b+r \sin \theta_{n} \\
& a_{0}=a+r, b_{0}=b \\
& a_{n+1}=a_{n}-\lambda b_{n}+\lambda b, 0 \leq n \leq n_{\theta}, n_{\theta}=2 / \lambda \\
& b_{n+1}=b_{n}+\lambda a_{n}-\lambda a \\
& a_{0}=a+r, b_{0}=b
\end{aligned}
$$
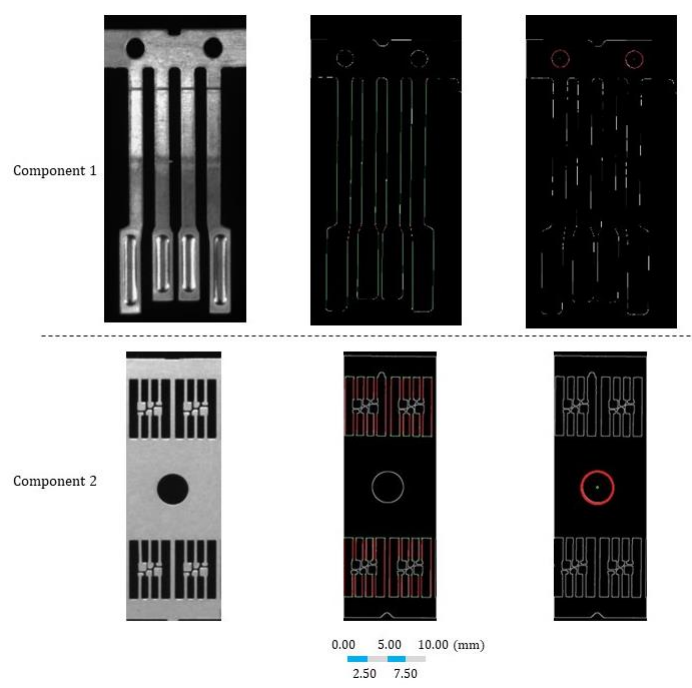

Fig.7. Defects location. From left to right are the original image, line location, and circular location. It is clear that the proposed method can accurately detect complicated lines and circles of various shapes.

The basic idea of the parallel property is to reduce the errors in the incremental calculation because the error has a great influence on the accuracy of the small size circular measurement in our study. As above, we introduced sub-intervals of the $\theta$ axis to obtain the parallel operation, as shown in (14), to make the same parameters as before. 


$$
\begin{aligned}
& x_{n+\frac{m g K}{M}}^{m}=a+r \cos \left(n+\frac{m \mathrm{~g} K}{M}\right), 0 \leq n<\frac{K}{M} \text { and } K=\frac{2 \pi}{\lambda} \\
& y_{n+\frac{m g K}{M}}^{m}=b+r \sin \left(n+\frac{m \mathrm{~g} K}{M}\right), 0 \leq m<M
\end{aligned}
$$

The results of the two types of metal components by applying the proposed method are shown in Fig. 7.

\section{Surface Defect Detection and Classification}

In the section, we proposed a new template-matching-based method by building a similarity measure function and introducing the improved pyramid search algorithm for online surface defect detection and classification. The advantages of the proposed method are summarized as follows: 1) The similarity measure is unchanged for non-linear illumination changes; 2) Segmentation without edge filtering; 3) When objects are partially visible or mixed with each other, the similarity measure is still accurate.

In the paper, the basic idea of the similarity measure function is to determine the similarity of the two objects, as shown in Fig. 8. Where arrows represent the gradient direction of objects $\mathrm{d}_{i}=\left(t_{i}, u_{i}\right)^{T}$ and template edge points $\mathrm{p}_{i}=\left(x_{i}, y_{i}\right)^{T}$, $i=1,2,3 \ldots, n$.The edge value is selected as the matching feature which can determine the final matching degree, the processing time, and final accuracy. The gradient direction values between the template and the edge of the object can be obtained by the edge extraction.

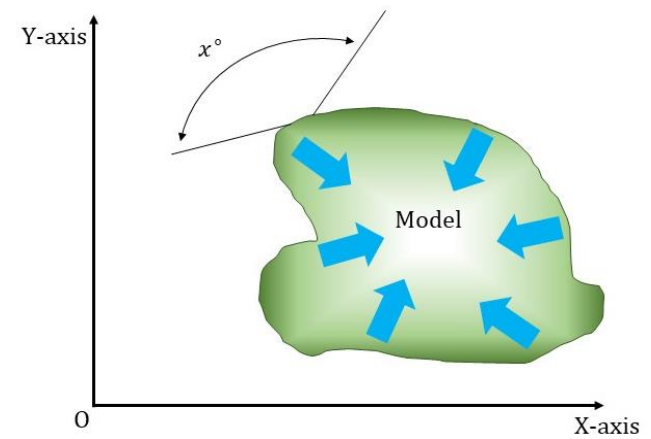

Fig.8. Similarity measure function

At the edge points of the object, the similarity measure function is shown as in (15). Where $d_{i}^{\prime}=R(\theta) d_{i}$ is transform the edge gradient vector in the template, $(x, y)$ is the edge point and $\mathrm{e}_{x, y}=\left(v_{x, y}, w_{x, y}\right)^{T}$ is the edge point direction vector.

$$
\mathrm{s}_{(x, y)}=\frac{1}{\mathrm{n}} \sum_{i=1}^{n}\left(d_{i}^{\prime}, e_{q+p^{\prime}}\right)=\frac{1}{\mathrm{n}} \sum_{i=1}^{n}\left(t_{i}^{\prime} v_{\left(x+x_{i}^{\prime}, y+y_{i}^{\prime}\right)}+u_{i}^{\prime} w_{\left(x+x_{i}^{\prime}, y+y_{i}^{\prime}\right)}\right)
$$

When the average value of the gradient vector dot product of the edge points is the largest, the gradient direction is the same direction; when the value is 0 , the gradient direction is vertical and orthogonal; when the negative value is the largest, the gradient direction is opposite. To determine the matching result more accurately, the above formula is normalized as follows. The function return value of 1 means a perfect matching, and that of 0 means no matching. The normalized similarity measure function is invariant to illumination.

$$
\mathrm{s}_{(x, y)}=\frac{1}{n} \sum_{i=1}^{n} \frac{\left(d_{i}^{\prime}, e_{q+p^{\prime}}\right)}{\left\|d_{i}\right\| \cdot\left\|e_{q+p^{\prime}}\right\|}=\frac{1}{n} \sum_{i=1}^{n} \frac{t_{i}^{\prime} v_{x+x_{i}, y+y_{i}^{\prime}}+u_{i}^{\prime} w_{x+x_{i}^{\prime}, y+y_{i}^{\prime}}}{\sqrt{t_{i}^{2}+u_{i}^{\prime 2}} \cdot \sqrt{v_{x+x_{i}, y+y_{i}}^{2}+w_{x+x_{i}^{2}, y+y_{i}^{\prime}}^{\prime}}}
$$

We set a threshold to remove the invalid similarity points for speeding up the search process, which is also a key parameter to terminate the similarity measure function operation. First, when the average value of the similarity measure is less than the minimum threshold, the search is stopped, the discriminant is shown in (17). In the process, parts of data points in template matching would be evaluated.

$$
\mathrm{s}_{(x, y)}>s^{\min }-1+\frac{m}{n}
$$

Additionally, the value of any point in the dataset is greater than the minimum threshold, namely, $\mathrm{s}_{m} \geq s_{\min } m / n$. When the previous data points are defective parts in the image, the average value would be very low, and the model would mistakenly consider it as an invalid matching. To solve this issue, we proposed a new discriminant by setting the coefficient $g$ in advance, as shown in (18). If $g=1$, strict termination conditions are adopted; if $g=0$, loose termination conditions are adopted. Through a large number of comparative tests, we usually set $g$ to 0.9 .

$$
\mathrm{s}_{m}<\min \left(\left(s^{\min }-1+\frac{1-g \cdot s^{\min }}{1-g} \cdot \frac{m}{n}\right),\left(s^{\min } \frac{m}{n}\right)\right)
$$

Considering the characteristics of the metal component, in our model, we proposed an improved pyramid-based algorithm to effectively speed up the model operation. Specifically, layer image base on Gaussian pyramid model to reduce complexity parameter $O(w h N n)$, where $w$ is width, $h$ is height, $N$ is the number of edge point of the template, and $n$ is the number of rotations of the template. The Gaussian pyramid can also be used to detect the structure of the image and obtain data more accurately by using Gaussian smoothing and subsampling. For example, the Gaussian data of the latter layer can be obtained from $f$ the previous layer by using our model. The Gaussian pyramid can span a large frequency range. This is because the Gaussian pyramid contains a series of low-pass filters that cut-off frequency gradually increases from the previous layer to the next layer by the difference 2 .

\section{EXPERIMENTS}

\section{A. Dataset collection}

Since most of the metal components in the real application are free of defects (industrial quality inspection must be conducted before products enter the market), it is difficult to obtain a large amount of experimental data. Currently, defect detection is aimed at specific production requirements without any established industry guidelines. In our study, we collected a dataset in the production line with the help of many researchers. Our dataset includes the following characteristics: 1) Two kinds of metal components, each containing 4 types of defect data, namely, scratch data, crack data, missing data, and needling data, as shown in Fig. 9; 2) The diameter of the defect data range from $0.2 \mathrm{~mm}$ to $2 \mathrm{~mm} ; 3$ ) Each type of defect metal components has 150 images, so a total of images is $2 \times(4 \times 150)=1200 ; 4)$ Considering the complex environmental conditions of the production line, including the different positions of the metal component and the non-uniform reflection properties of the surface. 


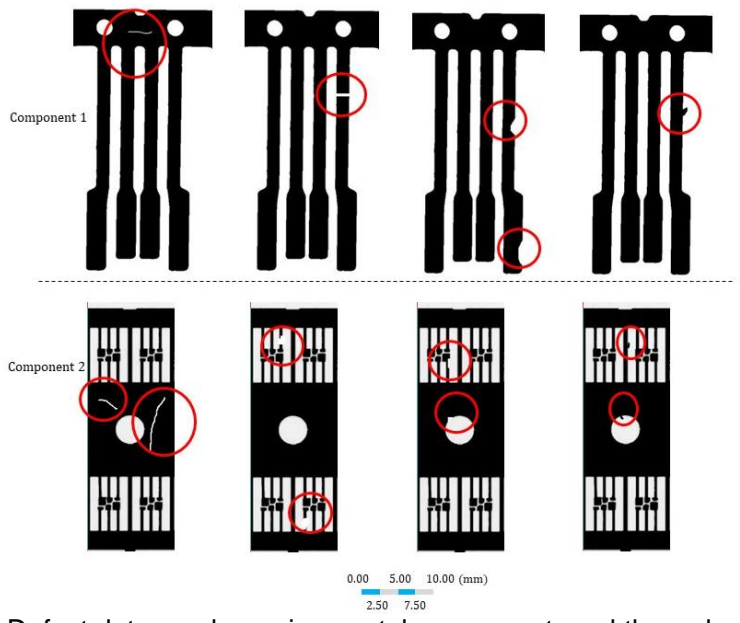

Fig.9. Defect data: each row is a metal component, and the red area is the defective part. From left to right are scratch data, crack data, missing data, and needling data.

\section{B. Dataset preprocessing}

Most of metal surface defect dataset are raw data with many types of noise, such as additive noise, multiplicative noise, quantization noise, and salt-and-pepper noise, which leads to many problems for detection model. To solve these problems, we proposed an adaptive-filtering-based data preprocessing method, which is mainly aimed at data noise caused by metal surface reflection and background impurities. Comparing with other popular methods, such as median-filtering-based methods, the proposed method has the following advantages: 1) Keeping more details or edge information in the image; 2) Correcting for the distortion in the data preprocessing; 3) Optimized for complex alt-and-pepper noise and quantization noise processing; 4) Speed up the processing operation.

The adaptive median filter window $S_{i, j}$ gives judging conditions by considering the changes in the median filter value $O_{\text {med }}$, the maximum filter value $O_{\max }$ and the minimum filter value $O_{\min }$. If the result of judging conditions is "yes", the current pixel value is replaced by the median value. Otherwise, the pixel value is not a noise point which can be retained. Specifically, following the steps: 1) if $O_{\min }<O_{\text {med }}<O_{\max }$, take the step 4); 2) if $O_{\min } \geq O_{\text {med }} \geq O_{\max }$, increase the size of filter window $S_{i, j}$; 3) If the size of $S_{m, n}$ is smaller than the size of $S_{\max }$, repeat step 1 , otherwise, output $\left.O_{\text {med }} ; 4\right)$ If $O_{\min }<O_{i, j}<O_{\max }$, output $O_{i, j}$; otherwise, output $O_{\text {med }}$. We selected the minimum filter window $3 \times 3$ and the maximum filter window $5 \times 5$.

As shown in Fig. 10, we show the results of data preprocessing by applying the proposed method. It is clear that data processed by the proposed method retains more detailed data and the edges are clearer.

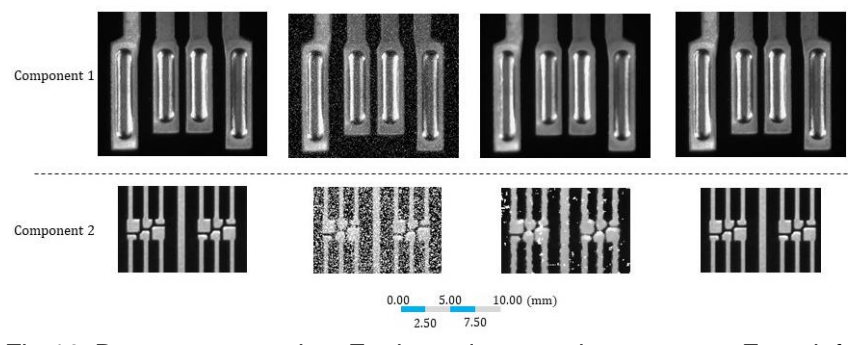

Fig.10. Data preprocessing. Each row is a metal component. From left to right are original image, the image with salt-and-pepper noise, median-filtering-based method, and the proposed method.

After removing the noise, we extracted the object from the complex background by using threshold segmentation. The basic idea is to divide the object and background into multiple areas by selecting multiple thresholds. To select the threshold accurately, we proposed a particle swarm-based method, as shown in equation (19). Where $\mathrm{V}_{i d}$ is the velocity of the id particle, and its range is $\left[-V_{\max d}, V_{\max d}\right] ; c_{1}, c_{2}$ is the learning parameter; $\operatorname{rand}()$ is the random number between 0 and $1 ; w$ is the inertia factor; $\mathrm{p}_{i d}(t)$ is the most valuable value found by the $t$-th particle; $x_{i d}$ is the current position of the $i$-th particle; $\mathrm{p}_{g d}$ is the optimal value found by the entire cluster; the range of the $d(1 \leq d \leq D)$ position is $\left[-X_{\max d}, X_{\max d}\right]$. Both optimal rang of the threshold are $T_{1} \in[131,154]$ and $T_{2} \in[175,790]$. After many comparative experiments, when $T_{1}=136, T_{2}=182$, we achieved the best image segmentation effect.

$$
\begin{aligned}
& \mathrm{V}_{i d}(t+1)= w \times \mathrm{V}_{i d}(t)+c_{1} \times \operatorname{rand}() \times\left[\mathrm{p}_{i d}(t)-x_{i d}(t)\right] \\
&+c_{2} \times \operatorname{rand}() \times\left[\mathrm{p}_{g d}(t)-x_{i d}(t)\right] \\
& x_{i d}(t+1)= x_{i d}(t)+v_{i d}(t+1) \\
& 1 \leq i \leq n \quad 1 \leq d \leq D
\end{aligned}
$$

The proposed method requires only a few parameters and can accelerate convergence. First, we constructed a 2D model by combining the gray value of the current image and the gray in the pixels and calculates the mean and variance. Additionally, we found the optimal gray value by calculating the optimal threshold. As shown in Fig. 11, we show the results of threshold segmentation by applying the proposed method.

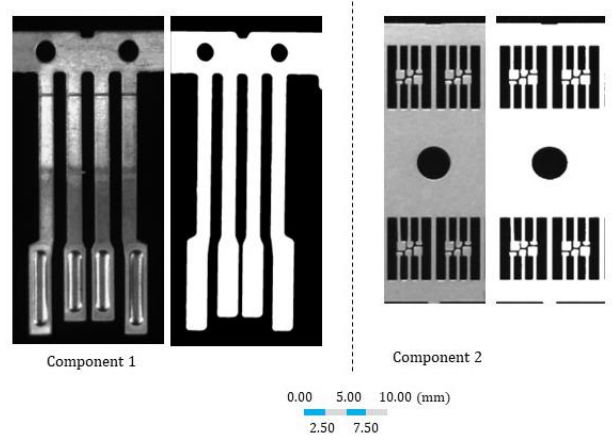

Fig. 11. Threshold segmentation. From left to right are original images, processed images.

\section{System design}

We built a defect detection system based on Opencv and Halcon library, the system structure is shown as in fig. 12. Each function of our system are shown as follow: 1) Device initialization, including device query and switch device; 2) 
Image acquisition, including continuous acquisition, trigger acquisition, start acquisition, stop acquisition, and soft trigger acquisition; 3) Image preservation, including BMP format and JPG format; 4) Parameter settings, including exposure, gain, frame rate, parameter acquisition; 5) Image preprocessing, as shown in Fig. 13, including image filtering, image enhancement, threshold segmentation, morphology, edge detection; 6) Measurement, as shown in Fig. 14, including the selection of different metal components and the display of measured data; 7) Defect detection, including defect location, defect area(pixel), defect type; 8) Results in display, including original images, template images, final results, as shown in Fig. 15.

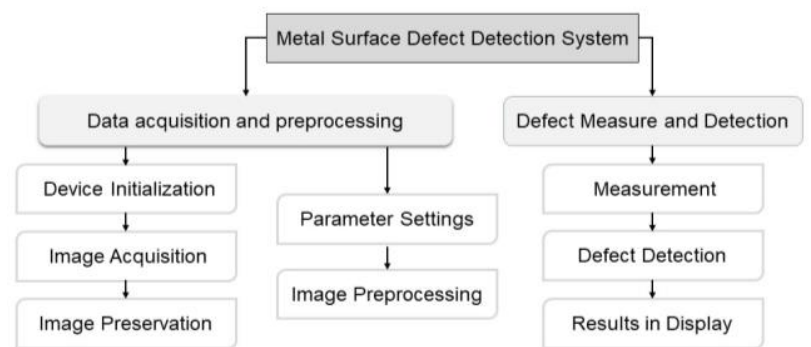

Fig. 12. System structure

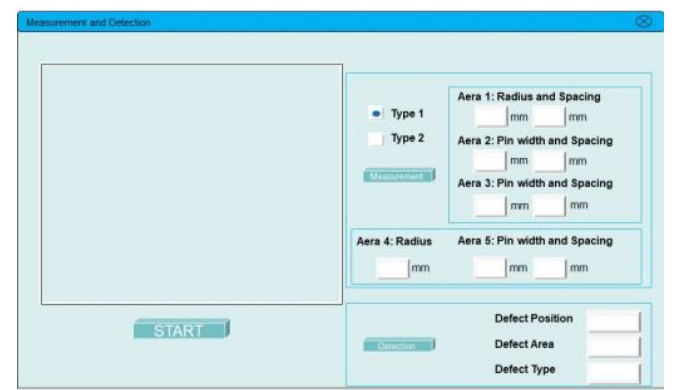

Fig. 13. Measurement and detection

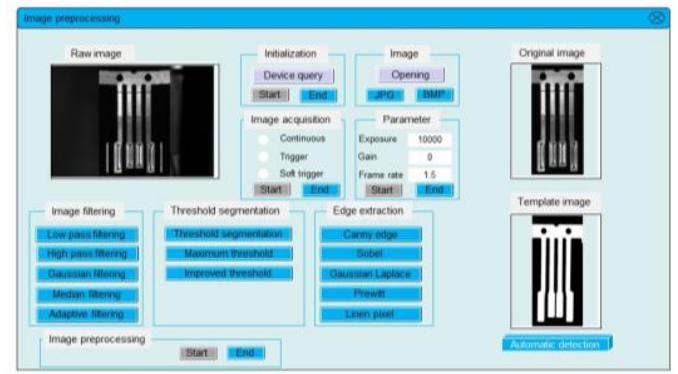

Fig.14. Image preprocessing

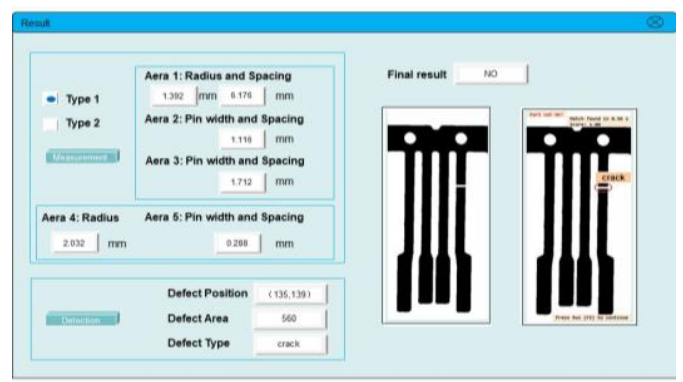

Fig.15. Final results

\section{Evaluation Metrics}

Motivated by the recent contribution evaluation methods of surface defect detection, in this paper we used the following evaluation metrics, as shown in (20): 1) False positive rate (FPR), namely, the proportion of pixels falsely detected as defects; 2) False negative rate (FNR), namely, the proportion of pixels falsely detected as non-defects; 3) Mean absolute error (MAE), namely, the difference between the detection result (DR) and ground truth (GT). Where FP is the number of pixels that are falsely detected as defects, $\mathrm{TN}$ is the number of non-defects pixels, $\mathrm{FN}$ is the number of undetected defective pixels, TP is the number of correctly detected defective pixels, $\mathrm{RN}$ is the row number, and $\mathrm{CN}$ is the column number. It is clear that the lower the values of FPR, FNR, and MAE, the better the result.

$$
\begin{aligned}
& F P R=\frac{F P}{F P+T N}, F N R=\frac{F N}{F N+T P}, \\
& M A E=\frac{1}{R N \times C N} \sum_{i=1}^{R N} \sum_{j=1}^{C N}|D R(i, j)-G T(i, j)|
\end{aligned}
$$

\section{E. Results and Analysis}

In this section, we conduct numerous experiments and evaluate the proposed method on two tasks, namely, detection rate and detection time. First, two sets of experiments were performed using our dataset, namely, testing the proposed method and deep learning-based models. Next, we used the public datasets to test the proposed method and compared it with other state-of-the-art results.

The proposed dataset: Four defects, including scratch, crack, missing, and needling. Five results are randomly selected from each type of defect. Detection results can be obtained, including the coordinates of the defect location, defect areas, running time, as shown in Table 1. The results show that the recognition rate of the proposed method on our dataset is $96.6 \%$, in which the deviation rate is less than $4 \%$, and the average detection time is 0.149 . After analyzing, we find that the main reason for the detection deviation is poor image preprocessing.

\begin{tabular}{|c|c|c|c|c|c|}
\hline \multicolumn{6}{|c|}{ RESULTS ON THE PROPOSED DATASET } \\
\hline Types & No. & Location $(\mathrm{x}, \mathrm{y})$ & Area(pixel) & Time(s) & Detection result \\
\hline \multirow{5}{*}{ scratch } & 1 & $(157,853)$ & 1309 & 0.156 & yes \\
\hline & 2 & $(325,301)$ & 560 & 0.153 & yes \\
\hline & 3 & $(617,305)$ & 1501 & 0.155 & yes \\
\hline & 4 & $(478,500)$ & 2078 & 0.155 & yes \\
\hline & 5 & $(525,489)$ & 460 & 0.153 & yes \\
\hline \multirow[t]{5}{*}{ crack } & 6 & $(846,465)$ & 1717 & 0.154 & yes \\
\hline & 7 & $(785,1621)$ & 2011 & 0.161 & yes \\
\hline & 8 & $(161,969)$ & 895 & 0.160 & yes \\
\hline & 9 & $(581,1261)$ & 956 & 0.159 & yes \\
\hline & 10 & $(557,157)$ & 784 & 0.159 & yes \\
\hline \multirow[t]{5}{*}{ missing } & 11 & $(105,1525)$ & 6417 & 0.159 & yes \\
\hline & 12 & $(177,1529)$ & 4561 & 0.159 & yes \\
\hline & 13 & $(105,1137)$ & 5414 & 0.160 & no \\
\hline & 14 & $(185,965)$ & 1354 & 0.162 & yes \\
\hline & 15 & $(157,465)$ & 2356 & 0.156 & yes \\
\hline \multirow[t]{5}{*}{ needling } & 16 & $(61,357)$ & 1254 & 0.156 & yes \\
\hline & 17 & $(625,569)$ & 2454 & 0.153 & yes \\
\hline & 18 & $(65,1261)$ & 564 & 0.160 & yes \\
\hline & 19 & $(225,1401)$ & 325 & 0.162 & yes \\
\hline & 20 & $(357,493)$ & 859 & 0.150 & yes \\
\hline
\end{tabular}

TABLE 1

However, the difference between the proposed method and deep learning-based models is unknown. Therefore, using our dataset to test several public deep learning-based models, the experimental results are shown in Table 2 . We consider the following state-of-the-art methods: 1) A Fast R-CNN was 
developed by introducing a Region Proposal Network which can reduce the running time [34]; 2) A Retina net was proposed for addressing the class imbalance, and the main idea is to reshape the cross-entropy loss [35]; 3) A new neural network named YOLO was proposed by dividing the detection framework into related class probabilities and spatially separated bounding boxes [36]. The results prove the effectiveness and superiority of the proposed method, especially in detection rate and detection time. This is because deep learning-based models cannot achieve excellent results with the insufficient training samples. In addition, due to a large number of parameters in most deep models, more running time must be required, which is a challenge for real-time detection. However, in most applications, since the products with defects cannot enter the market, a large amount of defect data would be difficult to collect.

TABLE 2

RESULTS ON DEEP LEARNING-BASED METHODS

\begin{tabular}{lll}
\hline \hline Method & Detection rate(\%) & Time(s) \\
\hline R-CNN & $83.8 \%$ & 0.163 \\
Retina Net & $91.7 \%$ & 0.179 \\
YOLO & $89.5 \%$ & 0.171 \\
The proposed model & $\mathbf{9 6 . 6 \%}$ & $\mathbf{0 . 1 4 9}$ \\
\hline \hline
\end{tabular}

The public dataset: We tested the proposed method on two popular public datasets, namely, Rail Surface Discrete Defect (RSDD) Dataset [37] and Magnetic Tile (MT) Blowhole Dataset [38]. Additionally, we consider the following state-of-the-art methods: 1) A contrast-adjusting method(CA) was extended by median-based Otsu's method for titanium-coated aluminum surface defect detection [39]; 2) A novel effective POFT-based model (SBD) was proposed by adjusting the input, that is, using the collection of multiple defective images as the input [40]; 3) A new method (SSD) for noisy image detection in the smooth background was proposed, which is aimed at image denoising and anomaly detection [41]. For fair comparisons, we use the popular evaluation metrics, namely, FPR, FNR and MAE.

Rail Surface Discrete Defect (RSDD) Dataset consists of two sub-datasets, and each has its own characteristics. Type 1 includes 67 defective images with more complicated background, which is used to test the ability of image preprocessing and edge detection. Type 2 includes 128 defective images with clear background, but the defects are more complicated, which can comprehensively test the performance of the proposed model.

TABLE 3

RESULTS ON THE RSDD DATASET

\begin{tabular}{lllll}
\hline \hline Type & Method & FPR & FNR & MAE \\
\hline 1 & CAT & 0.010 & 0.379 & 0.012 \\
& SBD & $\mathbf{0 . 0 0 4}$ & 0.312 & $\mathbf{0 . 0 0 7}$ \\
& SSD & 0.016 & $\mathbf{0 . 2 0 0}$ & 0.017 \\
2 & The proposed model & 0.007 & 0.297 & 0.010 \\
& CAT & 0.006 & 0.470 & 0.010 \\
& SBD & $\mathbf{0 . 0 0 3}$ & 0.444 & 0.008 \\
& SSD & 0.005 & 0.266 & 0.007 \\
& The proposed model & 0.004 & $\mathbf{0 . 2 3 7}$ & $\mathbf{0 . 0 0 7}$ \\
\hline \hline
\end{tabular}

The quantitative comparisons are listed in Table 3. It is clear that all the methods can successfully finish defect detection, and their low MAE means that the result is close to the real situation. For CAT, the key idea is to enhance the contrast of the image. Therefore, CAT has a higher missed detection when detects the images with a more complicated background, like the RSDD dataset. Similarly, for SBD, due to the effective phase filtering method, it can find the defect location more accurately. But SBD would filter out part of important features which can also lead to a higher missed detection. In contrast, SSD tends to detect defects more aggressively. Although it can finish defect detection in complex conditions, it has a higher false rate of detection. Considering the real applications, the proposed method achieves a more balanced performance and is more suitable for real applications. Specifically, for type 1 (images with more complicated background), all three evaluation metrics are lower than most other methods because of more effective edge detection and image preprocessing. For type 2 (image with more complicated defects), both FNR and MAE are the lowest that proves the correctness of each part in the proposed model.

MT Surface Defect Database consists of 115 defective images what is collected in different illumination. Especially, due to the randomness of the defective image collection, the dataset has a different size of defects, obvious noise, and severe vignette effect. Therefore, the MT dataset is a challenge for surface defect detection.

The quantitative comparisons are listed in Table 4. It is clear that the proposed method achieves state-of-the-art detection results. The main reason is that our improved image preprocessing method can eliminate multiple noises, which greatly reduces FPR and FNR. In contrast, CA and SSD falsely detected the unclear area of the edge that can increase FPR. Due to the effective edge detection method, SBD achieved a strong ability to detect defects in changing image size. But SSD cannot retain features in detail, which would result in a higher rate of missed detection.

TABLE 4

RESULTS ON THE MT DATASET

\begin{tabular}{llll}
\multicolumn{4}{c}{ RESULTS ON THE MT DATASET } \\
\hline \hline Method & FPR & FNR & MAE \\
\hline CAT & 0.022 & 0.304 & 0.023 \\
SBD & 0.007 & 0.487 & 0.009 \\
SSD & 0.017 & 0.445 & 0.018 \\
The proposed model & $\mathbf{0 . 0 0 5}$ & $\mathbf{0 . 2 3 1}$ & $\mathbf{0 . 0 0 6}$ \\
\hline \hline
\end{tabular}

\section{CONCLUSION}

In the paper, we proposed a novel model for automatic surface defects detection, which introduces the Gaussian operator and the improved Hough-based method. The model has a strong defect pixel point location performance and can classify defects with a faster speed. We achieved a mean precision of $96 \%$ to detect defects that are commonly presented in many applications and reduced the average time to $0.149 \mathrm{~s}$. Our results are superior to other deep learning-based models in terms of the recognition rate and the detection speed. To fully evaluate the proposed model, we tested our method on the public datasets and evaluated it by using three popular experiment metrics. The results showed that our method achieved a more balanced performance, which means that our method can well balance missed detection, false detection, and average error detection. Specifically, considering that complex defects are critical and need detection quickly, they may cause a more serious impact on product quality. Although our method achieved a lower recognition rate for defects detection in complex backgrounds, it can avoid misdiagnose.

In future work, we will improve the feature extraction by introducing the multiscale features segmentation method and 
the bionic mechanism, such as [42], [43] and [44]. Besides, we will collect more dataset with data preprocessing, which is expected to be a benchmark dataset for the metal surface defects detection.

\section{REFERENCES}

[1] Q. Liu, X. Fang, L. Liu, C. Yang and Y. Sun, "Automated Visual Defect Detection for Flat Steel Surface: A Survey," IEEE Transactions on Instrumentation and Measurement., vol. 69, no. 3, pp. 624-644, Mar. 2020.

[2] Y. Wang, F. Xie, S. Ma and L. Dong, "Review of surface profile measurement techniques based on optical interferometry," Optics and Lasers in Engineering., vol. 93, pp. 164-170, Jun. 2017.

[3] O.R. Real, M.J. Castro-Toscano, J.C. Rodríguez-Quiñonez, O. Serginyenko, D. Hernández-Balbuena, M. Rivas-Lopez et al., "Surface Measurement Techniques in Machine Vision: Operation, Applications, and Trends," in Optoelectronics in Machine Vision-Based Theories and Applications, Moises Rivas-Lopez, Oleg Sergiyenko, Wendy Flores-Fuentes and Julio Cesar Rodríguez-Quiñonez eds., Chicago, IL, USA: IGI Global, 2019, ch. 4, pp. 79-104. [Online]. Available: http://doi:10.4018/978-1-5225-5751-7.ch004

[4] D.M. Tsai, S.C. Wu, and W.Y. Chiu, "Defect detection in solar modules using ICA basis images," IEEE Transactions on Industrial Informatics, vol. 9, no. 1, pp. 122-131, Jul. 2012.

[5] K. Yildiz, A. Buldu, M. Demetgul, and Z. Yildiz, "A novel thermal-based fabric defect detection technique," The Journal of The Textile Institute, vol. 106, no. 3, pp. 275-283, May. 2014.

[6] C. Wang, N. Wang, S.C. Ho, X. Chen, and G. Song, "Design of a New Vision-based Method for the Bolts Looseness Detection in Flange Connections," IEEE Transactions on Industrial Electronics, vol. 67, no. 2, pp. 1366-1375, Feb. 2019.

[7] X. Li, H. Jiang, and G. Yin, "Detection of surface crack defects on ferrite magnetic tile," NDT and E International, vol. 62, pp. 6-13, Mar. 2014.

[8] M.R. Halfawy, and J. Hengmeechai, "Automated defect detection in sewer closed circuit television images using histograms of oriented gradients and support vector machine," Automation in Construction, vol. 38, pp. 1-13, Mar. 2014.

[9] J. Cao, J. Zhang, Z. Wen, N. Wang, and X. Liu, "Fabric defect inspection using prior knowledge guided least squares regression," Multimedia Tools and Applications, vol. 76, no. 3, pp. 4141-4157, Nov. 2015.

[10] Q. Zou, Z. Zhang, Q. Li, X. Qi, Q. Wang, and S. Wang, "DeepCrack: Learning Hierarchical Convolutional Features for Crack Detection," IEEE Transactions on Image Processing, vol. 28, no. 3, pp. 1498-1512, Oct. 2018.

[11] W. Sun, R. Zhao, R. Yan, S. Shao, and X. Chen, "Convolutional discriminative feature learning for induction motor fault diagnosis," IEEE Transactions on Industrial Informatics, vol. 13, no. 3, pp. 1350-1359, Feb. 2017.

[12] K. Yildiz, A. Buldu, and M. Demetgul, "A thermal-based defect classification method in textile fabrics with K-nearest neighbor algorithm," Journal of Industrial Textiles, vol. 45, no. 5, pp. 780-795, Oct. 2014.

[13] J. Sun, P. Wang, Y.K. Luo, and W. Li, "Surface defects detection based on adaptive multiscale image collection and convolutional neural networks," IEEE Transactions on Instrumentation and Measurement, vol. 68, no. 12, pp. 4787-4797, Mar. 2019.

[14] S.J. Chang, and J.B. Park, "Wire Mismatch Detection Using a Convolutional Neural Network and Fault Localization Based on Time-Frequency-Domain Reflectometry," IEEE Transactions on Industrial Electronics, vol. 66, no. 3, pp. 2102-2110, May. 2018.

[15] Z. Zuo, L. Yang, Y. Liu, F. Chao, R. Song and Y. Qu, "Histogram of Fuzzy Local Spatio-Temporal Descriptors for Video Action Recognition," IEEE Transactions on Industrial Informatics., vol. 16, no. 6, pp. 4059-4067, Jun. 2020.

[16] Y. Qu, G. Yue, C. Shang, L. Yang, R. Zwiggelaar and Q. Shen, "Multi-criterion mammographic risk analysis supported with multi-label fuzzy-rough feature selection," Artificial Intelligence in Medicine., to be published. DOI: 10.1016/j.artmed.2019.101722.

[17] X. Li, S.K. Tso, X.P. Guan, and Q. Huang, "Improving automatic detection of defects in castings by applying wavelet technique," IEEE
Transactions on Industrial Electronics, vol. 53, no. 6, pp. 1927-1934, Nov. 2006.

[18] M. Yazdchi, M. Yazdi, and A.G. Mahyari, "Steel surface defect detection using texture segmentation based on multifractal dimension," in 2009 International Conf. on Digital Image Processing, Bangkok, Thailand, 2009, pp. 346-350.

[19] N. Cai, G. Cen, J. Wu, F. Li, H. Wang, and X. Chen, "SMT solder joint inspection via a novel cascaded convolutional neural network," IEEE Transactions on Components, Packaging and Manufacturing Technology, vol. 8, no. 4, pp. 670-677, Jan. 2018.

[20] K. Yildiz, "Identification of wool and mohair fibres with texture feature extraction and deep learning," IET Image Processing, vol. 14, no. 2, pp. 348-353, Feb. 2020.

[21] B. Liu, Z. Ju, and H. Liu, "A structured multi-feature representation for recognizing human action and interaction," Neurocomputing, vol. 318, pp. 287-296, Nov. 2018.

[22] S.S. Martínez, J.G. Ortega, J.G. García, A.S. García, and E.E. Estévez, "An industrial vision system for surface quali0rty inspection of transparent parts," The International Journal of Advanced Manufacturing Technology, vol. 68, no. 5-8, pp. 1123-1136, Apr. 2013.

[23] F.P. Leon, and S. Kammel, "Inspection of specular and painted surfaces with centralized fusion techniques," Measurement, vol. 39, no. 6, pp. 536-546, Jul. 2006.

[24] F.J.C. Herrero, D.F. García, and R. Usamentiaga, "Inspection system for rail surfaces using differential images," IEEE Transactions on Industry Applications, vol. 54, no. 5, pp. 4948-4957, May. 2018.

[25] F.C. Chen, and M.R. Jahanshahi, "NB-CNN: Deep learning-based crack detection using convolutional neural network and Naïve Bayes data fusion," IEEE Transactions on Industrial Electronics, vol. 65, no. 5, pp. 4392-4400, Oct. 2017.

[26] L. R. Ramírez-Hernández, J. C. Rodríguez-Quiñonez, M. J. Castro-Toscano, D. Hernández-Balbuena, W. Flores-Fuentes, M. Rivas-López et al., "Stereoscopic Vision Systems in Machine Vision, Models, and Applications,". in Machine Vision and Navigation, O. Sergiyenko, W. Flores-Fuentes and P. Mercorelli eds., Cham, Zug, Switzerland: Springer, Cham, 2019, pp. 241-265. [Online]. Available: https://doi.org/10.1007/978-3-030-22587-2_8

[27] J.C. Rodríguez-Quiñonez, O. Sergiyenko, W. Flores-Fuentes, M. Rivas-lopez, D. Hernandez-Balbuena, R. Rascón et al., "Improve a 3D distance measurement accuracy in stereo vision systems using optimization methods' approach," Opto-Electronics Review, vol. 25, no. 1, pp. 24-32, May. 2017

[28] L. Lindner, O. Sergiyenko, J. C. Rodríguez-Quiñonez, M. Rivas-Lopez, D. Hernandez-Balbuena, W. Flores-Fuentes et al., "Mobile robot vision system using continuous laser scanning for industrial application," Industrial Robot., vol. 34, no. 4, pp. 360-369, Jun. 2016.

[29] G. Trujillo-Hernández, J. C. Rodríguez-Quiñonez, L. R. Ramírez-Hernández, M. J. Castro-Toscano, D. Hernández-Balbuena, W. Flores-Fuentes et al., "Accuracy Improvement by Artificial Neural Networks in Technical Vision System," in IECON 2019-45th Annual Conference of the IEEE Industrial Electronics Society, Lisbon, Portugal, Portugal, 2019, pp. 5572-5577.

[30] Y. Ai, S. P. Zhu, D. Liao, J.A.F.O. Correia, C. Souto, A.M.P. De jesus et al., "Probabilistic modeling of fatigue life distribution and size effect of components with random defects," International Journal of Fatigue, vol. 126, pp. 165-173, Sept. 2019.

[31] J.W. Gao, X.N. Pan, J. Han, S.P. Zhu, D. Liao, Y.B. Li et al., "Influence of artificial defects on fatigue strength of induction hardened S38C axles," International Journal of Fatigue., to be published. DOI: 10.1016/j.ijfatigue.2020.105746.

[32] S.P. Zhu, Y.Z. Hao and D. Liao, "Probabilistic modeling and simulation of multiple surface crack propagation and coalescence," Applied Mathematical Modelling., vol. 78, pp. 383-398, Feb. 2020.

[33] M. Valenzuela-Delgado, W. Flores-Fuentes, M. Rivas-López, O. Sergiyenko, L. Lindner, D. Hernández-Balbuena et al., "Electrolyte Magnetohydrondyamics Flow Sensing in an Open Annular Channel-A Vision System for Validation of the Mathematical Model," Sensors, vol. 18, no. 6, pp. 1683, May. 2018.

[34] S. Ren, K. He, R. Girshick, and J. Sun, "Faster r-cnn: Towards real-time object detection with region proposal networks," in Advances in Neural Information Processing Systems (NIPS), Montreal, Quebec, Canada, 2015, pp. 91-99.

[35] T.Y. Lin, P. Goyal, R. Girshick, K. He, and P. Dollar, "Focal loss for dense object detection," in Proc. of the IEEE International Conf. on Computer Vision (ICCV), Venice, Italy, 2017, pp. 2980-2988. 
[36] J. Redmon, S. Divvala, R. Girshick, and A. Farhadi, "You only look once: Unified, real-time object detection," in Proc. of the IEEE Conf. on Computer Vision and Pattern Recognition (CVPR), Las Vegas, NV, USA, 2016, pp. 779-788.

[37] J. Gan, Q. Li, J. Wang, and H. Yu, "A hierarchical extractor-based visual rail surface inspection system," IEEE Sensors Journal, vol. 17, no. 23, pp. 7935-7944, Oct. 2017.

[38] Y. Huang, C. Qiu, and K. Yuan, "Surface defect saliency of magnetic tile," The Visual Computer, vol. 36, no. 1, pp. 85-96, Aug. 2018.

[39] M. Win, A.R. Bushroa, M.A. Hassan, N.M. Hilman, and A. Ektessabi, "A contrast adjustment thresholding method for surface defect detection based on mesoscopy," IEEE Transactions on Industrial Informatics, vol. 11, no. 3, pp. 642-649, Mar. 2015.

[40] X. Bai, Y. Fang, W. Lin, L. Wang, and B. Ju, "Saliency-based defect detection in industrial images by using phase spectrum," IEEE Transactions on Industrial Informatics, vol. 10, no. 4, pp. 2135-2145, Sept. 2014.

[41] H. Yan, K. Paynabar, and J. Shi, "Anomaly detection in images with smooth background via smooth-sparse decomposition," Technometrics, vol. 59, no. 1, pp. 102-114, Jan. 2017.

[42] J. Yu, H. Gao, W. Yang, Y. Jiang, W. Chin, N. Kubota et al., "A discriminative deep model with feature fusion and temporal attention for human action recognition," IEEE Access, vol. 8, pp. 43243-43255, Mar. 2020.

[43] Z. Ju, X. Ji, J. Li, and H. Liu, "An integrative framework of human hand gesture segmentation for human-robot interaction," IEEE Systems Journal, vol. 11, no. 3, pp. 1326-1336, Sept. 2015.

[44] Z. Ju, G. Ouyang, M. Wilamowska-Korsak, and H. Liu, "Surface EMG based hand manipulation identification via nonlinear feature extraction and classification," IEEE Sensors Journal, vol. 13, no. 9, pp. 3302-3311, Apr. 2013.

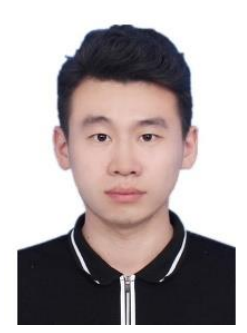

Jiahui Yu received his B.S. and M.S. degrees in intelligent systems from Shenyang Ligong University, China, in 2017 and 2019, respectively. Since 2019, he is currently working toward the Ph.D. degree at the University of Portsmouth, U.K. He has published over 10 papers in journals and conference proceedings. His current research interests include machine intelligence, pattern recognition, and human-robot/computer interaction and collaboration.

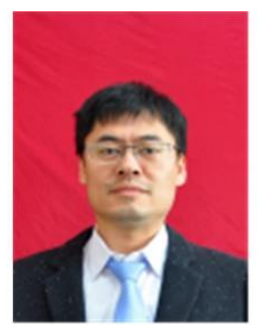

Hongwei Gao received his Ph.D. degree in the field of pattern recognition and intelligent system from Shenyang Institute of Automation (SIA), Chinese Academy of Sciences (CAS) in 2007. Since September 2015, he has been a professor of School of Automation and Electrical Engineer-ing, Shenyang Ligong University. Currently, he is the leader of academic direction for optical and electrical measuring technology and system. His research interests include digital image processing and analysis, stereo vision and intelligent computation. He has published more than sixty technical papers in these areas as first authors or co-authors.

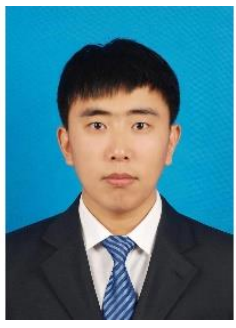

Jian Sun received his B.S. and M.S. degrees in control engineering from Shenyang Ligong University, China, in 2016 and 2019, respectively. Since 2019, he is currently working toward the Ph.D. degree at Shenyang Ligong University, China. His current research interests include machine learning, digital image processing, pattern recognition, and signal processing.

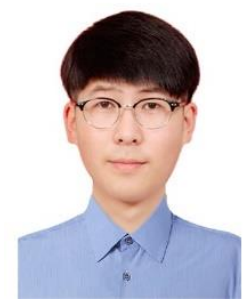

Wei Yang was born in Heilongjiang, China, in 1994. He received his B.S. degree in automation from Shenyang Ligong University of China in 2016 and his M.S. in automation and electrical engineering: detection technology and automatic equipment from Shenyang Ligong University. His research interests include deep learning, pattern recognition and digital image processing.

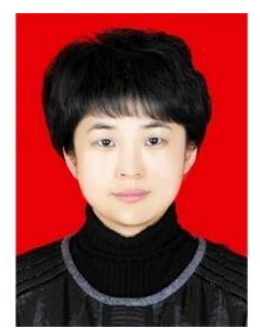

Yueqiu Jiang received the B.S. and M.S. degrees in computer science from Shenyang Ligong University, China, in 1998 and 2001, respectively, and the Ph.D. degree in computer science from Northeast University, China, in 2004. She worked as a lecturer from March 2004 to Aug. 2006 in School of Science, Shenyang Ligong University, and worked as an associate professor form Sep. 2006 to Aug. 2010 in School of Science, Shenyang Ligong University. Currently, she is a professor in School of Information Science and Engineering, Shenyang Ligong University. Her research interests include image processing, multimedia applications and satellite communications and signal processing.

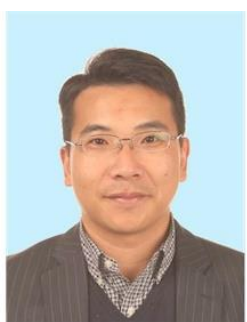

Zhaojie Ju (M'08-SM'16) received the B.S. degree in automatic control and the M.S. degree in intelligent robotics from the Huazhong University of Science and Technology, China, and the Ph.D. degree in intelligent robotics from the University of Portsmouth, U.K. He held research appointments at University College London, London, U.K., before he started his independent academic position at the University of Portsmouth, in 2012. He has authored or coauthored over 180 publications in journals, book chapters, and conference proceedings and received four Best Paper Awards and one Best AE Award in ICRA2018. His research interests include machine intelligence, pattern recognition and their applications on human motion analysis, multi-fingered robotic hand control, human-robot interaction and collaboration, and robot skill learning.

Dr. Ju is an Associate Editor of several journals, such as IEEE TRANSACTIONS ON CYBERNETICS, IEEE TRANSACTIONS ON COGNITIVE AND DEVELOPMENTAL SYSTEMS and Neurocomputing. 